\title{
FCR System in Hokkaido University Hospital
}

\author{
Eiichi Asai, Masao Takano, and Masami Chida
}

The complete digital $x$-ray system using FCR is now being used in daily diagnosis as a part of the picture archiving and communications system (PACS) in Hokkaido University (HU) Hospital. This project is based on a 3 year plan started in 1988. In the first year (1988), six Fuji Computed Radiography (FCR) units (two FCR501. one FCR502, three FCR7000), PACS interfaces, and an optical disk system were installed. In the first year, about $60 \%$ of $x$-ray filming changed from the conventional technique to the FCR, and $60 \%$ of FCR images were transferred to PACS. In the second year, we began to use FCR in gastrointestinal (GI) study with two additional FCR7000s and three $x$-ray TV units. Then the percentage of plain films made by FCR increased to $80 \%$. In the third year, angiographic studies will be converted to FCR. A specially designed built-in type FCR is under development to examine the entire spine. When this project is complete, almost all conventional radiography except chest tomography will be performed by FCR. The tomography $x$-ray computed radiography (CR) unit with automatic-imaging plate (IP) changer is expected to be developed soon.

Copyright (C) 1991 by W.B. Saunders Company

KEY WORDS: FCR, PACS, digital imaging, computed radiography.

$\mathbf{W}^{\mathrm{n}}$ E PRESENTED THE computed radiography (CR) imaging system to the world for the first time at the International Congress of Radiology in Brussels, Belgium in 1981. It was recognized as a new technology: digital radiography. The FCR 101 was introduced to the market in 1983, and the FCR201 and the FCR901 were introduced in 1985 . These early units could not digitize all $\mathrm{x}$-ray filming in the hospital, however, because of their size and throughput.

The third-generation model (FCR7000) marketed in April 1988 was designed on the basic concept of "decentralized imaging plate (IP) processing," and was characterized by a marked reduction in the size of the device and a well-prepared interface. This enables the entire

From the Equipment Products Division, Fuji Photo Film Company, and Department of Radiology, Hokkaido University Hospital.

Address reprint requests to Eiichi Asai, Senior Research Staff, Fuji Photo Film Company Ltd, Miyanodai Technology Development Center, 798, Miyanodai Kaisei-machi, Ashigarakami Kanagawa 256, Japan.

Copyright (C) 1991 by W.B. Saunders Company

0897-1889/91/0404-0104\$03.00/0 radiographic image to be fully converted into FCR in a practical sense. In recent years, there has been an upsurge in the introduction of PACS, which has prompted more and more medical facilities to choose full installation of FCR with an eventual eye to more effective use of a digital radiographic images (eg, filing and CRT diagnosis), thereby achieving substantial results.

In 1988, Hokkaido University (HU) Hospital decided to convert all its $x$-ray filming into FCR, using a 3-year plan with the goal of introducing total PACS. We describe the system configurations and features.

\section{OUTLINE OF THE FCR SYSTEM IN HU HOSPITAL}

The building housing the radiology department of HU Hospital was constructed in 1965. As a result, the $x$-ray room layout has several negative factors, such as a lack of separation of patient and worker passages. The system operation rooms were not centralized and were located next to each x-ray room. In addition, considerable effort had previously been made to introduce a daylight-type conveyer system to transport films between the filming room and the film processor. Therefore, we had to avoid any lessening of efficiency owing to the replacement with FCR. To overcome these problems, doctors, technicians, and engineers in charge of this project met for a series of talks on preliminary arrangements to thrash out an optimal system design.

Table 1 shows the maximum and average number of $x$-ray films generated at HU each day. These data show that (1) the maximum amount of films is about twice as many as are produced by the average hospital so that we need a margin in the performance of the system; (2) to digitize all x-ray filming, we need more than 10 FCRs to handle the maximum load; (3) additional engineering effort should be made to develop a special FCR for whole spine x-ray; and (4) careful investigation is expected in angiographic study because of its strong demand for processing speed.

Figure 1 is a schematic of the system configuration that resulted from the 1988 and 1989 installations. In $1988,60 \%$ of radiographic images 
Table 1. Number of X-Ray Images Made Daily

\begin{tabular}{llcr}
\hline $\begin{array}{l}\text { X-ray } \\
\text { Room }\end{array}$ & \multicolumn{1}{c}{ Purpose } & $\begin{array}{r}\text { Films/Day } \\
\text { (Maximum) }\end{array}$ & $\begin{array}{r}\text { Films/Day } \\
\text { (Average) }\end{array}$ \\
\hline 1 & Chest, abdomen, W.S. & 140 & \\
$1^{\prime}$ & Chest, abdomen & 140 & \\
2 & Spine, pelvis, thigh & 180 & \\
3 & Cervical, chest, abdomen & 120 & \\
5 & Breast, arm, leg & 170 & $650^{*}$ \\
P & Portable & 50 & \\
H & Head & 100 & \\
T-1 & Bone tomography & 90 & \\
T-2 & Chest tomography & 160 & 50 \\
U & Urinary tract & 80 & \\
U-TV & Urinary tract & 80 & $70 \dagger$ \\
TV-1 & Gastrointestinal tract & 150 & \\
TV-2 & & 120 & \\
TV-3 & & 150 & $180 \dagger$ \\
TV-4 & 120 & \\
AOT1 & Blood vessel & 250 & \\
AOT2 & & 300 & $210 \neq$ \\
Total & & & 1,160 \\
\hline * First year. & & \\
† Second year. & & \\
† Third year. & &
\end{tabular}

were produced by FCR; in 1989, it increased up to $80 \%$.

\section{System Layout}

Filming of a patient in the standing position, mainly of chests and abdomens, is completely automated with two FCR501 units. Likewise, filming of the spine (thoracic and lumbar spine) is performed with the FCR502. In the operation of these units, technicians are freed from the work of handling the IP, so that they can devote themselves to positioning the patient and to other patient care issues. Because the cervical spine $x$-ray requires six or seven images for each examination and the time allotted for changing the patients' position is short, a magazine-type IP changer for standing position is used in this technique. Furthermore, one FCR 7000 reader is installed in this area and is used for pediatric radiography, hip joint, femur, and other regions.

Radiography of the bones of the extremities and mammography is performed in the same floor block, and one FCR 7000 reader is used exclusively to process this workload. These two reading units functionally overlap and have the additional role of providing a backup to breakdowns of the other units.

Both the head radiography room and bone tomography room are located at the other end of the radiology department. Because more than 100 images are generated in these rooms, another FCR7000 reader was installed for exclusive use in both rooms. The images taken at bedside in the ward also are processed with this reader.

In 1989, gastrointestinal (GI) study, urinary tract study, and other studies with contrast media were changed to FCR with three IP type $\mathrm{x}$-ray TV units and two FCR7000 readers. At the end of 1989, there were eight FCR7000 readers in all and the percentage of FCR images had increased to $80 \%$.

Three image recorders were installed together at the same location to facilitate water supply and to streamline the film delivery.

By fully capitalizing on the features enabling decentralized installation, we built a total digitized radiography system using FCR which systematically links the $\mathrm{x}$-ray rooms and film processing room located in three separate places. This experience taught us that a new FCR7000 system could be adequately set up even in a hospital handicapped by varied installation restraints.

\section{ID System}

The magnetic card used to input the patient's identification (ID: name, number, sex, and date of birth) to the FCR system is automatically issued by the terminal of the host computer (NEC hospital information system, HIS). This prevents mistakes and improves work at the reception desk of the radiology department.

Two additional information items are also recorded in the magnetic card. The first item is a control flag used to distinguish whether or not to send images to PACS. The HIS computer knows this information, because it can be determined by the department requesting the examination. The other item is an order number successively issued by HIS to identify the examination itself.

The ID terminals read these data in the magnetic card and send them to the FCR image reader. We took special care to reduce the work flow by placing 12 ID terminals near the $x$-ray rooms. Because these terminals are interconnected, information can be correctly transferred to the specific FCR image reader independent of the ID terminal used for registration. 


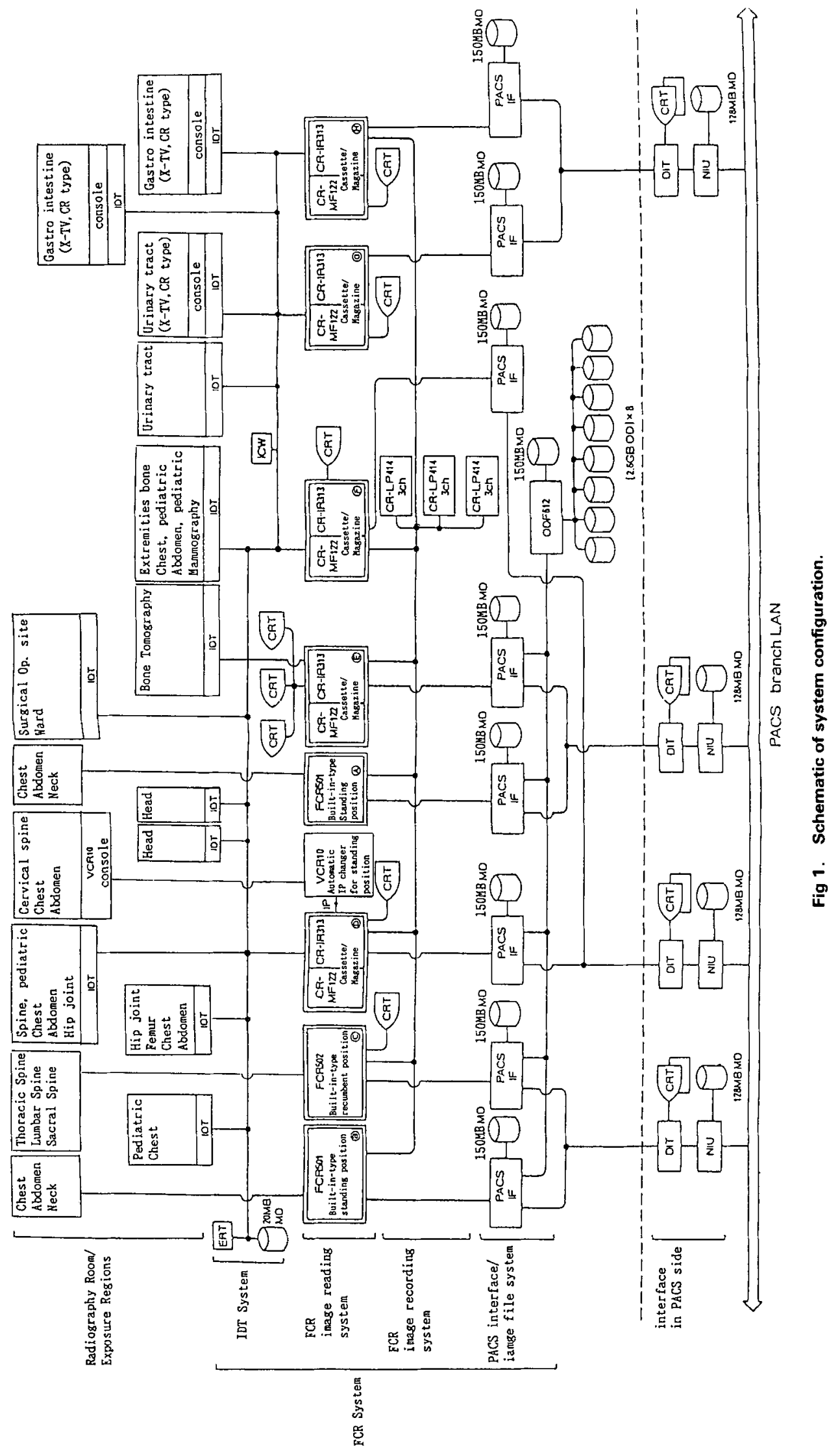




\section{PACS Interface and Image File for FCR}

The image data read out by FCR is automatically sent to the PACS side in real time through the PACS interface unit. This unit features several intelligent functions.

1. Automatic transmission of data is assured by sorting out the transmission $\mathrm{ON} / \mathrm{OFF}$ flag for PACS assigned to each image.

2. A magnetic disc buffer automatically retransmits the data without interrupting the FCR reading unit even if a transmission error occurs to the PACS side.

3. The image is sent to the FCR optical disk file unit simultaneously with the transmission to PACS.

4. Manual transmission from the magnetic disk buffer to the PACS side, to the FCR image reader side for film-making purposes, and to the optical disk side is allowed.

The FCR image file unit is an entity separate from the PACS side file. It is connected to eight reading units, thereby permitting recording of all images produced by the individual reading units.
This type of installation prevents accidents such as loss or cancellation of important medical data.

Because this image file allows the setting of reversible compression (about one half) and irreversible compression (about one twelfih) for each exposure menu, it is able to record data in the optical disk by matching the compression rate to the required image quality. It also houses a 150Mbyte magnetic disk capable of controlling up to 1.2 million recorded images. This allows the required image to be selected easily by the ID number and other data.

At present, PACS covers only three departments: the outpatient orthopedic department, the outpatient room for first admission of patients to the hospital (general medicine), and the radiology departments. Currently it uses write-once-type optical disks; therefore, we cannot reuse disks. Thus, we wish to initiate a careful study of the possibility of introducing a magento-optical disk unit that provides erasing and rerecording functions so that we can match the expansion of the PACS side system. 